\title{
The Pusher as an Object of the Simulation Model in the Tasks of Studying Cyclic Mechanisms Dynamics
}

\author{
Viktor V. Telegin ${ }^{1, a}$ and Igor V. Telegin ${ }^{1, b^{*}}$ \\ ${ }^{1}$ Lipetsk State Technical University, Lipetsk, Russia \\ avv.telegin@yandex.ru, bigor.v.telegin@gmail.com
}

Keywords: Pusher, Cyclic Mechanism, Dynamic Processes, Object-Oriented Technologies

\begin{abstract}
The cyclic mechanism is one of the basic automatic machines components. It is the cyclic mechanisms that define the performance and reliability limits of these machines. The methods for assessing the automatic machines reliability are based on data of dynamic forces acting on the mechanism's links, leading to their deformation and possible destruction under certain operating conditions. Simulation modeling of dynamic processes in cyclic mechanisms is based on its representation in the form of interconnected typical objects, the allowable properties and limits of which are known. The article presents the dynamics study results one of the basic high-speed automatic machines mechanism in the object-oriented representation. Mechanism performs translational motion and allows the possibility of one-way kinematic connection breaking.
\end{abstract}

\section{Introduction}

Any mechanism can be considered as a collection of some elements, interconnected by various kinds of connections. Through these links, the elements of the mechanism interact with each other, thus ensuring its functioning $[1,2]$. The term element will denote a single mechanism detail, its fragment, or, conversely, several details at once. This definition of an element corresponds to the concept of an object, one of the basic in the component modeling theory of complex dynamic systems and object-oriented technologies [3, 4].

For a visual representation of mechanisms, schemes (principle, kinematic, hydraulic, pneumatic and others), flat and three-dimensional drawings, as well as dynamic models are used. Let us juxtapose each element of one mechanism with an object, according to the term given above. Obviously, this object can contain as one single detail or even a fragment of it, or several details. Any processes in such an object can be modeled in any way and, in accordance with the principles of encapsulation and polymorphism, at this stage, their nature and description, mathematical, software or physical, is not considered.

This approach is of interest both when solving problems associated with the development of dynamic and mathematical mechanisms models, and, which is especially important, their software implementation for further computer processing $[5,6]$.

\section{Mathematical model of the pusher object}

A pusher is a translationally moving link, for example, the cam mechanism pusher of an ejector from the dies (Fig. 1) of a cold stamping machine (CSM) for nuts manufacture [7, 8]. The pusher object provides the possibility of one-way kinematic connection breaking with the object preceding it (Fig. 1, positions 1 and 2). 


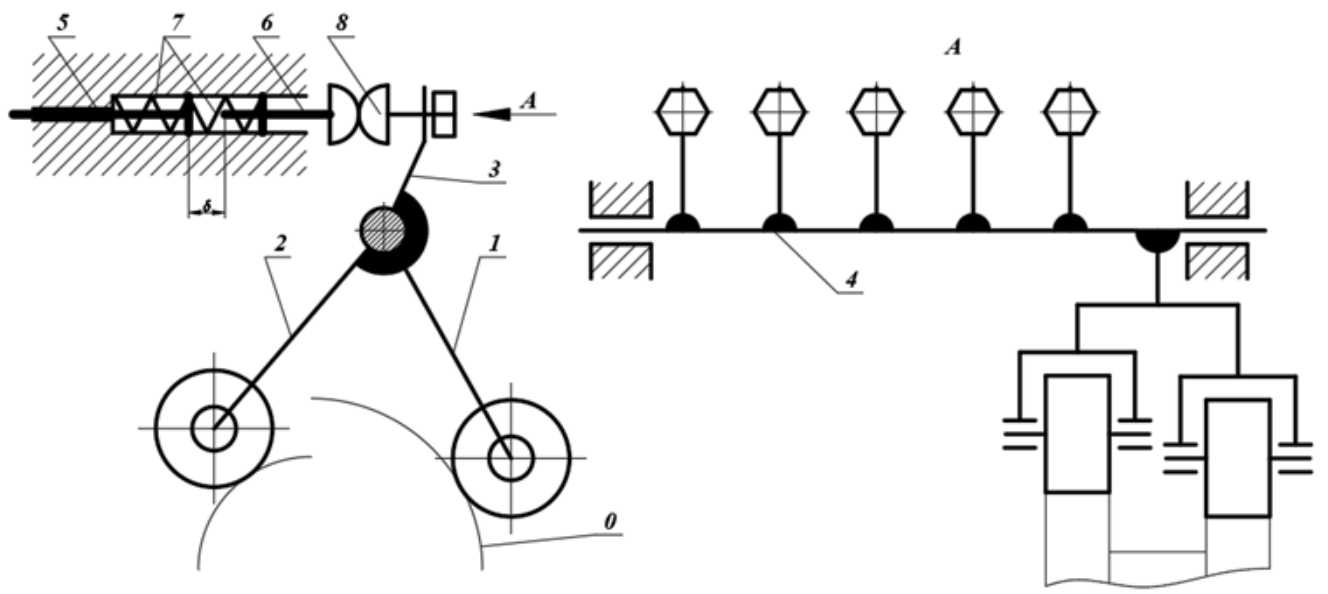

Fig. 1. Kinematic diagram of the CSM ejection mechanism:

$\mathbf{0}$-block of cams, 1, 2-forward and reverse levers, 4-axle, 5, 6-parts of a composite ejector rod, 7 -springs, 8 -adjusting screw.

A conventional image, the object itself and its model are shown in Fig. 2 and 3.

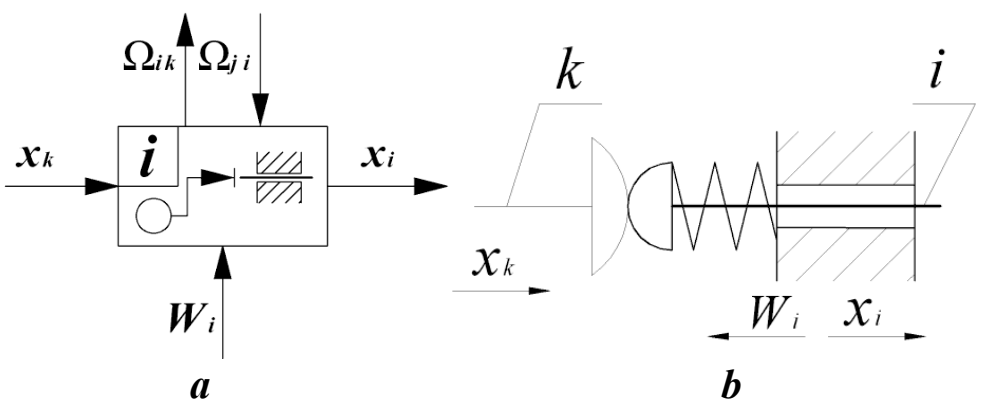

Fig. 2. Pusher object: a-object model (i-th object), b-kinematic diagram.

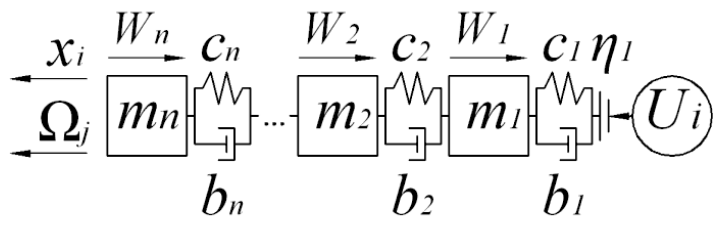

Fig. 3. The dynamic model of the pusher object.

Masses motion equations of the object dynamic model [3]: 


$$
\left\{\begin{aligned}
m_{1} \ddot{x}_{1}= & -c_{1}\left(x_{1}-U_{i}\left(x_{k}\right)\right)-b_{1}\left(\dot{x}_{1}-U_{i}^{\prime}\left(x_{k}\right) \dot{x}_{k}\right)+ \\
& +c_{2}\left(x_{2}-x_{1}\right)+b_{2}\left(\dot{x}_{2}-\dot{x}_{1}\right)+W_{1} \\
m_{2} \ddot{x}_{2}= & -c_{2}\left(x_{2}-x_{1}\right)-b_{2}\left(\dot{x}_{2}-\dot{x}_{1}\right)+ \\
& +c_{3}\left(x_{3}-x_{2}\right)+b_{3}\left(\dot{x}_{3}-\dot{x}_{2}\right)+W_{2} \\
\ldots \quad \ldots \quad & \ldots \\
m_{n} \ddot{x}_{n}= & -c_{n}\left(x_{n}-x_{n-1}\right)-b_{n}\left(\dot{x}_{n}-\dot{x}_{n-1}\right)+W_{n}+\Omega j
\end{aligned}\right.
$$

In Eq. 1, as in Fig. 3, the parameter $\boldsymbol{x}_{\boldsymbol{k}}$ is not necessarily linear. For example, if the object corresponds to a cam disc mechanism with a linear follower, $\boldsymbol{x}_{\boldsymbol{k}}$ is the cam angle. Rigidity $\boldsymbol{c}_{\boldsymbol{1}}$ is a piecewise linear function, the value depends on the size of the gap $\boldsymbol{\eta}_{1}$.

\section{Stability of the pusher object model}

The main parameters that determine the object behavior under the dynamic force influence are its mass and stiffness. The currently used 3D modeling methods in the design of mechanical engineering products $[9,10,11]$ allow to be calculated these parameters with a high degree of accuracy. The object under study is adequate to the real link of the mechanism if, within the specified limits of its parameters variation, the values of the criteria for its stability are close when using models of varying complexity in calculations $[12,13]$.

In Fig. 4 shows the details of the CSM ejection mechanism (see Fig. 1, positions 5, 6) and the tested object, $20 \mathrm{~mm}$ in diameter and $200 \mathrm{~mm}$ length. The object mass is $\boldsymbol{m} \approx 0.49 \mathrm{~kg}$, the rigidity is $\boldsymbol{c} \approx 3.3 \cdot 10^{8} \mathrm{~N} / \mathrm{m}$ (density of the steel: $\boldsymbol{\rho} \approx 7800 \mathrm{~kg} / \mathrm{m}^{3}$, Young's modulus $\boldsymbol{E} \approx \mathbf{2 . 1} \cdot 10^{11} \mathrm{~N} / \mathrm{m}^{2}$ ).

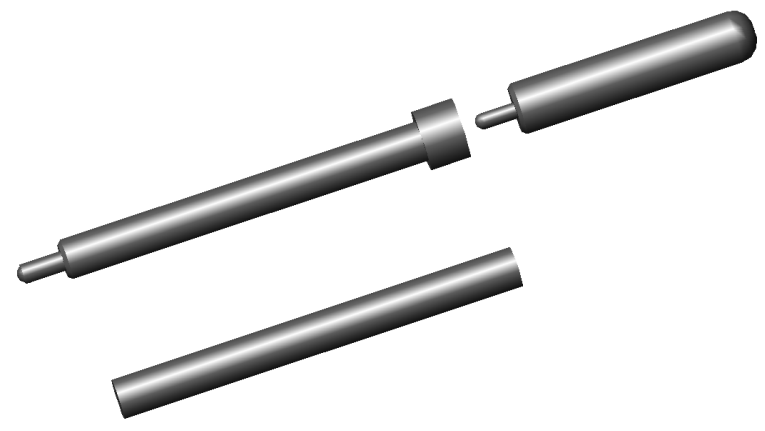

Fig. 4. Test object and details of the CSM ejection mechanism.

The maximum speed of the model output link and the accuracy of its positioning $\boldsymbol{\delta}_{\max }$ are taken as criteria for the object stability. The parameters of the model varied within the following limits: weight $-0.05 \ldots 1.5$, rigidity $-10^{8} . . .4 \cdot 10^{9}$.

We will assume that the movement of the link is carried out according to a harmonic law with amplitude equal to $\mathbf{0 . 0 1} \mathrm{m}$, which, in particular, corresponds to the stroke of the CSM ejector. The pusher movement speed $(\boldsymbol{\omega})$ is assumed equal to 10 strokes per second (600 strokes per minute), closing load $1000 \mathrm{~N}$, dissipation coefficient $\boldsymbol{\psi}=\mathbf{0 . 4}$.

$$
\begin{aligned}
& U=0.01 \cdot \sin 2 \pi \omega t, \\
& U^{\prime}=0.01 \cdot \cos 2 \pi \omega t .
\end{aligned}
$$

The calculation data carried out on models of varying complexity (single-mass, dual-mass, and ten-mass) in the Maple software $[14,15]$ turned out to be practically the same (coincidence in the 
sixth decimal place). Fig. 5-6 show the calculated dependences of the objects kinematic parameters and positioning accuracy.
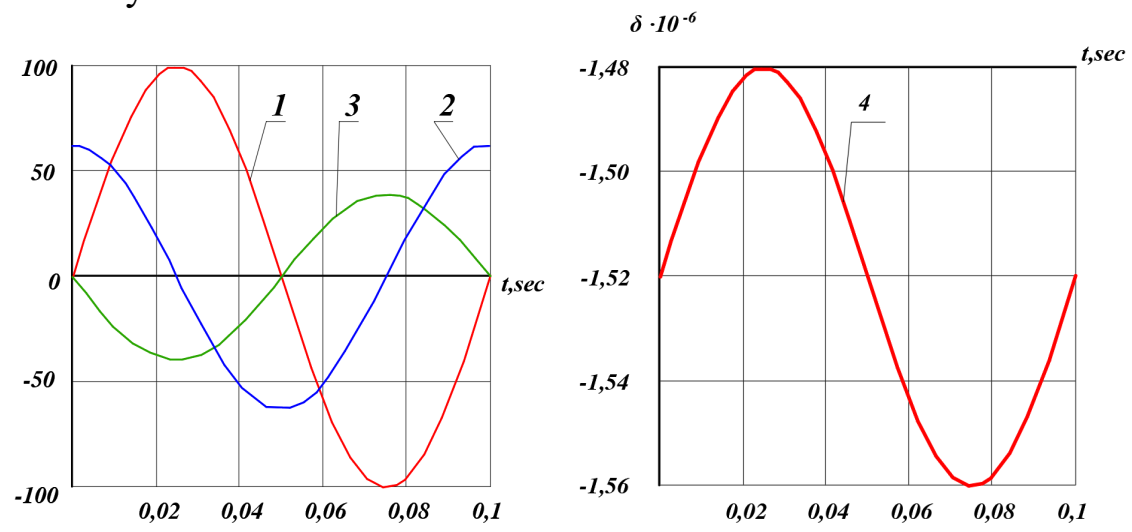

Fig. 5. Kinematic characteristics of the object: 1 -displacement $\left(10^{-4} \mathrm{~m}\right)$, 2 - speed $\left(10^{-2} \mathrm{~m} / \mathrm{sec}\right), 3$ - acceleration $\left(1 \mathrm{~m} / \mathrm{sec}^{2}\right), 4$-positioning accuracy $(1 \mathrm{~m})$.

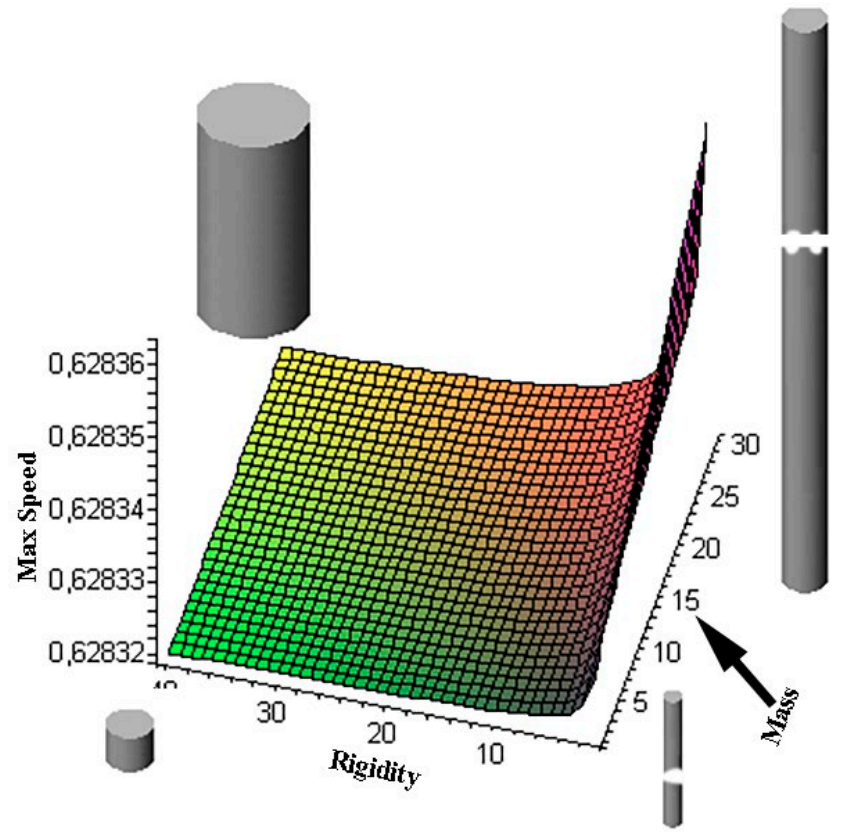

Fig. 6. To calculate the stability of an object: $\boldsymbol{\omega}=\mathbf{1 0}, \boldsymbol{\psi}=\mathbf{0 . 4}, \boldsymbol{P}=1000 \mathrm{~N}$.

It should be noted that the influence of the dissipation coefficient on the behavior of this object is insignificant. At the same time, the elastic-inertial parameters of the object noticeably affect the dynamic processes occurring in the object in a mode when a break in the kinematic chain of the object with the previous link is possible. This situation is shown in Fig. 7. The closing force in this case is $20 \mathrm{~N}$. At the same time, there is a significant difference in the calculation results obtained for the single-mass and dual-mass models. The calculation results obtained on the basis of the twomass and ten-mass models are close (the difference is about $10 \%$ ). 


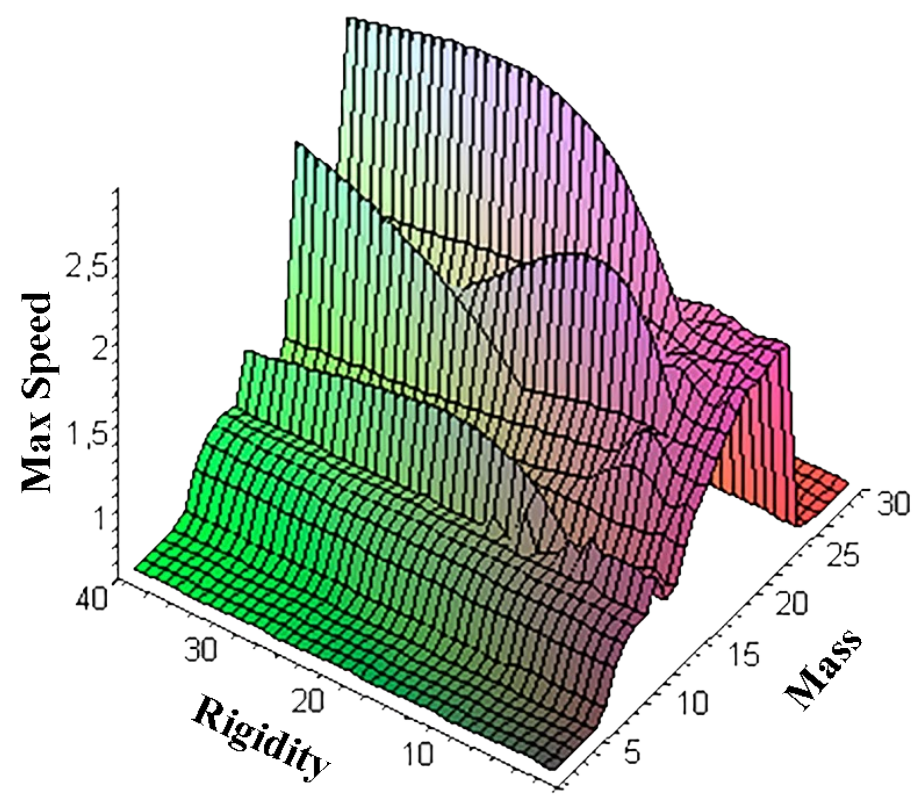

Fig. 7. To the calculation of the object stability. Ten-mass model: $\boldsymbol{\omega}=\mathbf{1 0}, \boldsymbol{\psi}=\mathbf{0 . 4}, \boldsymbol{P}=\mathbf{2 0} \mathrm{N}$.

\section{Conclusion}

A technique is proposed for assessing the limits of objects applicability, from which a simulation model of a mechanical system (mechanism) is formed.

The software implementation of this technique can be used as an integral part of software systems for the dynamic analysis of mechanical systems, which are based on the component modeling methods of object-oriented technologies.

\section{References}

[1] I.I. Wolfson, Oscillations of Machines with Cyclic Action Mechanisms, Mashinostroenie, Leningrad, 1990.

[2] 2. V.L. Weits, I. A. Gidaspov, G.V. Tsaryov, Dynamics of Drives with Closed Kinematics, Publishing House of Mordovia University, Saransk, 1991.

[3] I. Telegin, A. Kozlov, A. Zhirkov, Component simulation in problems of calculated model formation of automatic machine mechanisms, MATEC Web Conf. International Conference on Modern Trends in Manufacturing Technologies and Equipment (ICMTMTE 2017). 129 (2017) 03016. https://doi.org/10.1051/matecconf/201712903016

[4] V. Telegin, A. Kozlov, T. Shumilova, Development and research of the rotating lever object as a dynamic model of a cycle mechanism, International Conference on Modern Trends in Manufacturing Technologies and Equipment (ICMTMTE 2018). 224 (2018) 02078. https://doi.org/10.1051/matecconf/201822402078

[5] V.V. Telegin, Performance Assessment in Design of High-Speed Automatic Machines Based on Analysis of Their Dynamics, Proceedings of the 6th International Conference on Industrial Engineering (ICIE 2020), Lecture Notes in Mechanical Engineering. (2021) 1000-1007. https://doi.org/10.1007/978-3-030-54814-8_115 
[6] I.V. Telegin, Dynamics of the crank press, RU Patent 2013618511. (2013).

[7] Yu.A. Miropolsky, Cold Die Forging on Automatic Machines, Mechanical Engineering, Moscow, 2001.

[8] G.A. Navrotsky, Forging and stamping machines, Mechanical engineering, Moscow, 1965.

[9] V.G. Kontsevich, Solid State Modeling of Engineering Products in Autodesk Inventor, DMK Press, Moscow, 2007.

[10]A.A. Alyamovsky, Engineering Computations in SolidWorks Simulation, DMK Press, Moscow, 2010.

[11] V.V. Telegin, A.M. Kozlov, A.V. Kirichek, Solid Modeling in Autodesk Inventor at Initial Stage of Training of Specialists in Field Mechanical Engineering, Proceedings of the 4th International Conference on Industrial Engineering (ICIE 2018), Lecture Notes in Mechanical Engineering. (2019) 1241-1247. https://doi.org/10.1007/978-3-319-95630-5_130

[12] A.V. Ryabova, V.Yu. Tertychny-Dauri, Elements of the theory of stability, Textbook, ITMO University, St. Petersburg, 2015.

[13] A.S. Volmir, Stability of deformable systems, Part 1, textbook for universities, Yurayt Publishing House, Moscow, 2021.

[14] V.Z. Aladiev, V.K. Boyko, E.A. Rovba, Programming in Maple and Mathematica Packages: Comparative Aspect, Monograph, Grodno State University, Grodno, 2011.

[15] Ch.G. Edwards, D.E. Penny, Differential Equations and Boundary Value Problems, Modeling and Computation with Mathematica, Maple and MATLAB, Dialectics-Williams, Kiev, 2007. 